\title{
Tumor Necrosis Factor Receptor Superfamily Member EDAR
}

National Cancer Institute

\section{Source}

National Cancer Institute. Tumor Necrosis Factor Receptor Superfamily Member EDAR. NCl Thesaurus. Code C159363.

Tumor necrosis factor receptor superfamily member EDAR (448 aa, $\sim 49 \mathrm{kDa}$ ) is encoded by the human EDAR gene. This protein is involved in signaling that modulates the development of ectodermal structures. 